\title{
Signal Separation and Reconstruction Method for Simultaneously Received Multi-System Signals in a Unified Wireless System
}

\author{
Takayuki Yamada, Doohwan Lee, Hiroyuki Shiba, Yo Yamaguchi, and Kazuhiro Uehara \\ NTT Network Innovation Laboratories, NTT Corporation \\ 1-1 Hikarinooka, Yokosuka-shi, Kanagawa, 239-0847 Japan \\ E-mail: yamada.takayuki@lab.ntt.co.jp
}

\begin{abstract}
These days, a wide variety of wireless systems are used and new wireless systems appear one after another on the market. In this regard, we proposed a unified wireless system that we call "flexible wireless system". Comprising flexible access points and a flexible signal processing unit, it collectively receives a wideband spectrum that includes multiple signals from various wireless systems. In cases of simultaneous multiple signal reception, however, reception performance degrades due to the interference among multiple signals. To address this problem, we propose a new signal separation and reconstruction method for spectrally overlapped signals. The method analyzes spectral information obtained by the short time Fourier transform to extract amplitude and phase values at each center frequency of overlapped signals. Using these values enables signals from received radio wave data to be separated and reconstructed for simultaneous multi-system reception. Computer simulation results obtained in evaluating the method's performance confirmed its effectiveness.
\end{abstract}

Keywords: Flexible Wireless System, signal separation, signal reconstruction, short-time Fourier transform

\section{INTRODUCTION}

A wide variety of wireless systems are used to enjoy ubiquitous network services. As information and communication services become more sophisticated and diversified, we are likely to see an explosion in the number of sensors and radio-frequency identification (RFID) terminals [1-2] in use. However, the increased diversity and number of wireless systems also cause problems such as inter-system interference and a lack of locations for suitable base station installation. These problems will become more severe in the future because wireless systems need to support an enormous number of wireless terminals.

These changes in wireless radio environments require a unified platform that can flexibly deal with various wireless radio systems. Therefore, we proposed a "Flexible Wireless System" (FWS) that uses software defined radio technology and cognitive radio technology. It receives radio waves including multiple signals from various wireless systems at distributed access points, stores all radio wave data in a network, and processes them by software [3]. Thus, as opposed to conventional wireless systems, the received radio waves are not processed at an access point but at a central station with rich storage and processing power

In FWS, signals from multiple systems may be spectrally overlapped because they are simultaneously received at a single access point. If received signals do not overlap, they can be separated by a conventional band pass filter (BPF). If they do overlap, however, the reception performance degrades because they interfere with each other. To obtain the desired signals from among various interfering signals, a highly accurate signal separation technology is required for simultaneously received multi-system signals. Many such technologies make use of multiple antennas; these include independent component analysis based approaches [4-5] and adaptive array approaches [6]. These methods are effective when the number of signals is less than or equal to the number of antennas. They cannot be applied, however, when it is assumed that the number of simultaneously received signals is more than the number of antennas on access points. A timefrequency masking approach has been proposed as a technology that is suitable for such cases [7-9]. This is a signal separating approach based on the sparsity of signals in both the time domain and the frequency domain (the timefrequency domain). However, this approach cannot be applied in cases when signals overlap in the time-frequency domain because the sparsity of the signals becomes low in such cases. Therefore, it is difficult to apply this approach when signals are overlapping under the time-frequency domain. To address this problem, we propose a new signal separation and reconstruction method for use in this case. This method separates spectrally overlapped signals through a simple procedure under the assumption that the amplitude and phase components of a variety of modulated signals are constant when clipping wave data with a short time window.

The rest of the paper is organized as follows. Section II describes FWS in detail. Section III explains our proposed signal separation method. In Section IV, we evaluate its performance through computer simulation. Section V concludes the paper with a brief summary of the main points.

\section{FLEXIBLE WIRELESS SYSTEM}

\section{A. Concept}

Figure 1 shows a conceptual diagram of FWS [3]. It is composed of flexible access points and flexible signal processing units. Both are connected via an access network, i.e., an optical fiber network. Each flexible access point 


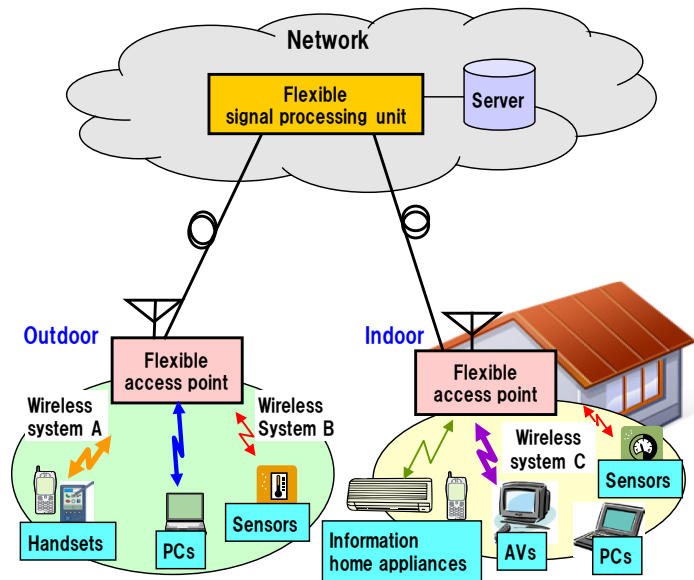

Figure 1. Conceptual diagram of FWS

consists of a broadband antenna, a broadband RF circuit, and an $\mathrm{AD} / \mathrm{DA}$ converter. The system can send and receive the radio waves of a wide variety of wireless systems. The flexible signal processing unit is attached to the network and processes the signal data by software. This architecture allows flexible and quick support for new wireless protocols because the protocols are processed by software. A statistical multiplexing effect can also be incorporated into FWS, enabling it to centralize many functions normally executed individually at a large number of base stations. As a similar concept with radio on fiber (ROF), this architecture is proposed in the "Fiber optic networks for distributed and extendible heterogeneous radio architectures" (FUTON) project [10]. The object of the FUTON project is to achieve broadband wireless transmission and inter-cell interference cancellation for fourth generation (4G) systems. The main difference between this research work and ours is that FWS supports transmission and reception in a wide variety of systems simultaneously.

Many challenges had to be met in developing FWS. For example, the flexible access points require equipment with wider and more diverse bandwidths [11] and an expanded dynamic range to achieve highly flexible transmission and reception. In radio wave data transfer between the flexible access points and the flexible signal processing unit, efficient data compression is required to allow the transfer of quite voluminous radio wave data sampled at a high sampling rate with high resolution. In the flexible signal processing unit, various types of signal processing techniques, including adaptive modulation and demodulation, and signal combination and separation, are needed to achieve simultaneous multisystem transmission and reception.

\section{B. Signal processing of the flexible signal processing unit}

The flexible signal processing unit has to reduce reception performance degradation caused by simultaneously received multi-system signals at the flexible access points. This is because radio wave data transferred from the flexible access points includes mixed signals of multiple wireless systems and noise over wider bandwidths. Received signals also include signals transmitted from wireless terminals, e.g., many kinds of RFID terminals that have no interference avoidance function as a means of access control. Therefore, co-channel interference among both identical and different system types increases. Moreover, differences in received signal levels increase not only due to the near-far problem but also due to the differences in transmission power among different systems. Furthermore, the receiver's noise level is high because it is generally difficult to achieve lower noise levels in broadband or multiband receiving equipment than in conventional equipment for a single system. This means that signal separation and noise reduction are very important techniques in signal processing. These techniques make it possible to achieve high performance levels in modulation classification, demodulation, and decoding in the receiving of multi-system signals.

\section{Signal processing scheme with stored radio wave data for simultaneously received multi-system signals}

To incorporate the above-mentioned techniques into the flexible processing unit, we are considering adopting a signal processing scheme with stored radio waves for simultaneously received multi-system signals. This scheme is one application that can make use of one of the main advantages of FWS. This advantage is that the radio waves, which include signals transmitted from a wide variety of wireless systems with various frequencies, are received at distributed access points, transferred without signal processing, stored in a network, and processed collectively with software. A network contains high performance computing servers with huge storage capacity that a general access point cannot have due to constraints on cost and installation location. Processing signals in a network makes it possible to use the network's rich variety of resources and apply networking technologies such as cloud computing technology. Therefore, the scheme can use a huge amount of information pertaining to time, frequency, and space domains.

\section{SignAl SEPARATION AND RECONSTRUCTION METHOD}

This section proposes a new signal separation method for signal processing, in which stored radio wave data is utilized for simultaneously received multi-system signals.

\section{A. Purpose \& Characteristics}

Since FWS simultaneously receives multiple signals at multiple bands, inter-system interference often occurs. If the receiver uses only a conventional BPF, the reception performance will be degraded. To solve this problem, we propose a signal separation and reconstruction method that separates the received multiple signals simultaneously.

As mentioned in the "Introduction" section, FWS has to separate simultaneously received signals even if the number of signals exceeds that of antennas and the signals overlap in the time-frequency domain. Therefore, we propose a method for separating spectrally overlapped signals under the assumption that the amplitude and phase components of a variety of modulated signals are constant when clipping wave data with a short time window. By doing this, desired signals' spectrum forms are determined by the window. Therefore, our method can separate multiple desired signal components from one signal received on a single antenna and reconstruct desired signals from the separated components. On problem is that 
signal separation by frequency domain becomes complex and difficult when signals with various symbol lengths are overlapped in a time domain. To solve this problem, our method uses wave data that is clipped by a shorter time window than the shortest symbol length among signals. Simulation analysis results have shown that the best window size is half of the symbol length, as we reported in [12].

This method we propose here separates spectrally overlapping signals of different center frequencies, regardless of the modulation method, by using the center frequencies and symbol rates of multiple signals. We have used it to sample received radio wave data at a sampling rate much higher than the symbol rate of various single systems for broadband receiving.

\section{B. Proposed method}

In this section, we introduce our proposed method for signal separation. The method separates signals by means of three steps, i.e., analysis, separation, and reconstruction, following the received radio wave data model and the assumption given below.

\section{B.1 Received radio wave data model}

An equation of received radio wave data, $y(t)$, is given by

$$
\begin{aligned}
& y(t)=\sum_{k=1}^{K} y_{k}(t)+n(t) \\
& y_{k}(t)=h_{k}(t) a_{k}(t) e^{j\left(\omega_{k} t+\phi_{k}(t)\right)}
\end{aligned}
$$

where $y_{k}(t)$ is the $k$-th $(k=1 \ldots \mathrm{K})$ signal component in time $t$, $a_{k}(t)$ and $\phi_{k}(t)$ are the amplitude and phase components of the $k$-th signal in time $t, h_{k}(t)$ is the channel impulse response or filter coefficient of the $k$-th signal, $\omega_{k}\left(=2 \pi f_{k}\right)$ is the center frequency of the $k$-th signal, and $n(t)$ is the noise component in $t$.

\section{B.2 Assumption}

Here, we define an assumption that each signal's amplitude and phase components are constant when clipping wave data with a short time window. The time length of the short time window is much shorter than the symbol length to eliminate the effect of symbol length differences. The equation for this assumption is given by

$$
\begin{aligned}
y_{k}(t, \tau) & =w(t-\tau) h_{k}(t) a_{k}(t) e^{j\left(\omega_{k} t+\phi_{k}(t)\right)} \\
& =w(t-\tau) A_{k, \tau} e^{j\left(\omega_{k} t+\Phi_{k, \tau}\right)}
\end{aligned}
$$

where $w(t)$ is the short time window function, $\tau$ is a time shift parameter for the function, $A_{k, \tau}$ and $\Phi_{k, \tau}$ are constant amplitude and phase components of the $k$-th signal in clipping with the short time window, and $A_{k, \tau}$ and $\Phi_{k, \tau}$ includes the averaged amplitude and phase fluctuation caused by $h_{k}(t)$.

\section{B.3 Procedure}

1) Analysis step
In order to use the above assumption, our method uses the short time window to analyze the frequency domain information of received radio waves. That is, in this step, the short-time Fourier transform (STFT) is executed. This step is shown in Fig.2. The STFT is a time-frequency analysis method in which fast Fourier transform (FFT) is executed sequentially by clipping wave data with a time-shifted short time window.

The wave data equation after the STFT is executed is given by

$$
\begin{aligned}
Y(\omega, \tau) & =\int_{-\infty}^{\infty} w(t-\tau) y(t) e^{-j \omega t} d t \\
& \approx \int_{-\infty}^{\infty} w(t-\tau)\left\{\sum_{k=1}^{K} h_{k}(t) a_{k}(t) e^{-j\left(\omega-\omega_{k}\right) t} e^{j \phi_{k}(t)}\right\} d t
\end{aligned}
$$

Note that the noise component is omitted here and below for simplicity in the derivation. However, the noise component is also considered in the performance evaluation. From Eq. (3), Eq. (4) becomes as follows:

$$
Y(\omega, \tau) \approx \sum_{k=1}^{K} A_{k, \tau} e^{j \Phi_{k, \tau}} \int_{-\infty}^{\infty} w(t-\tau) e^{-j\left(\omega-\omega_{k}\right) t} d t .
$$

These Eqs. (4) and (5) represent the spectrum of received wave data clipped with a short time window shifted by $\tau$.

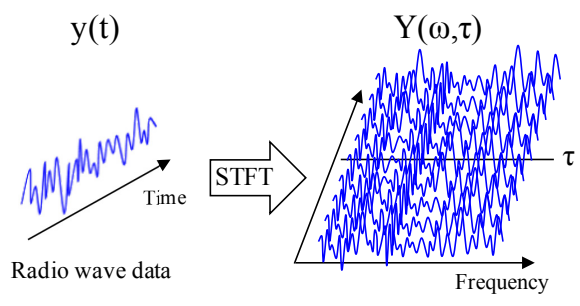

Figure 2. Analysis step

\section{2) Separation step}

In this step, the amplitude and phase components of spectrum overlapped signals are separated. This step is shown in Fig.3 when two signals, sig.1 and sig.2, are overlapping. Here, we assume that the center frequencies are obtained from a prior information source such as a network database, or as a result of signal detection. The amplitude and phase values on the center frequencies of overlapped signals are extracted from the spectrum of received wave data, and Eq. (5) becomes as follows:

$$
\begin{aligned}
{\left[\begin{array}{c}
Y\left(\omega_{1}, \tau\right) \\
\vdots \\
Y\left(\omega_{K}, \tau\right)
\end{array}\right] \approx } & {\left[\begin{array}{c}
\sum_{k=1}^{K} A_{k, \tau} e^{j \Phi_{k, \tau}} \int_{-\infty}^{\infty} w(t-\tau) e^{-j\left(\omega_{1}-\omega_{k}\right)} d t \\
\vdots \\
\sum_{k=1}^{K} A_{k, \tau} e^{j \omega_{k, \tau}} \int_{-\infty}^{\infty} w(t-\tau) e^{-j\left(\omega_{K}-\omega_{k}\right) t} d t
\end{array}\right] } \\
& \approx\left[\begin{array}{ccc}
\int_{-\infty}^{\infty} w(t-\tau) d t & \cdots & \int_{-\infty}^{\infty} w(t-\tau) e^{-j\left(\omega_{1}-\omega_{K}\right) t} d t \\
\vdots & \ddots & \vdots \\
\int_{-\infty}^{\infty} w(t-\tau) e^{-j\left(\omega_{K}-\omega_{1}\right) t} d t & \cdots & \int_{-\infty}^{\infty} w(t-\tau) d t
\end{array}\right]\left[\begin{array}{c}
A_{1, \tau} \tau^{j \Phi_{1, \tau}} \\
\vdots \\
A_{K, \tau} e^{j \Phi_{K, \tau}}
\end{array}\right]
\end{aligned}
$$

Here, we show the three parts of Eq. (6) as follows:

$$
\begin{aligned}
Y(\tau) & =\left[\begin{array}{llll}
Y\left(\omega_{1}, \tau\right) & \cdots & Y\left(\omega_{K}, \tau\right)
\end{array}\right]^{\mathrm{T}} \\
G(\tau) & =\left[\begin{array}{ccc}
\int_{-\infty}^{\infty} w(t-\tau) d t & \cdots & \int_{-\infty}^{\infty} w(t-\tau) e^{-j\left(\omega_{1}-\omega_{K}\right) t} d t \\
\vdots & \ddots & \vdots \\
\int_{-\infty}^{\infty} w(t-\tau) e^{-j\left(\omega_{K}-\omega_{1}\right) t} d t & \cdots & \int_{-\infty}^{\infty} w(t-\tau) d t
\end{array}\right]
\end{aligned}
$$


$X(\tau)=\left[\begin{array}{lll}A_{1, \tau} e^{j \Phi_{1, \tau}} & \cdots & A_{K, \tau} e^{j \Phi_{K, \tau}}\end{array}\right]^{\mathrm{T}}$

where $Y(\tau)$ and $X(\tau)$ are the extracted value vector and the separated component vector in $\tau,[]^{\mathrm{T}}$ shows a transposed vector, and $G(\tau)$ is the base signal matrix in $\tau$, which is generated by the center frequency of the overlapped signals and the window function. When the overlapped signals are two signals that are sent from each single carrier systems $(\mathrm{K}=$ 2), $G(\tau)$ is a 2 by 2 matrix.

Therefore, this step calculates $X(\tau)$ by using $Y(\tau)$ and $G(\tau)$. From Eqs. (7), (8), and (9), Eq. (6) becomes as follows:

$$
Y(\tau)=G(\tau) X(\tau)
$$

Subsequently $X(\tau)$ in Eq. (10) is given by

$$
X(\tau)=G^{-1}(\tau) Y(\tau)
$$

where $G^{-1}(\tau)$ is the inverse matrix of $G(\tau)$. This inverse matrix always exists if every center frequency of the overlapped signals is different under the frequency resolution of FFT.

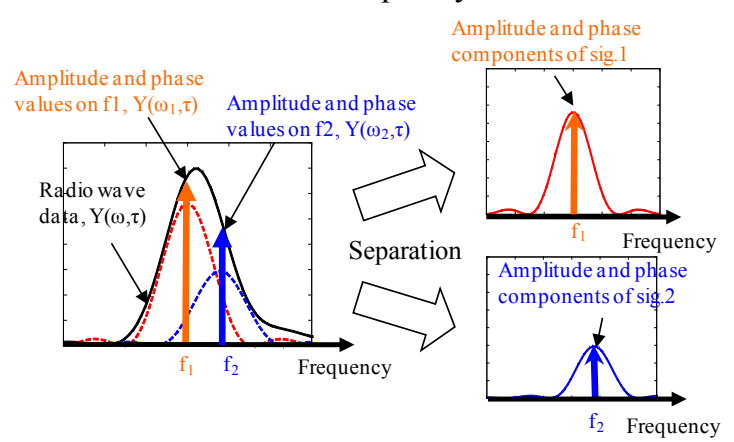

Figure 3. Separation step

\section{3) Reconstruction step}

In this step, overlapped signals are reconstructed individually by the separated amplitude and phase components in Eq. (11). Individual signals clipped with the short time window are recovered by substituting these separated components and center frequencies into Eq. (3). Overlapped signals are reconstructed by combining them. The reconstructed signal $y_{k}^{\prime}(t)$ is given by

$$
y_{k}^{\prime}(t)=\sum_{\tau} y_{k}^{\prime}(t, \tau)=\sum_{\tau} w(t-\tau) A_{k, \tau}^{\prime} e^{j\left(\omega_{k} t+\Phi_{k, \tau}^{\prime}\right)}
$$

Where $A_{k, \tau}^{\prime}$ and $\Phi_{k, \tau}^{\prime}$ are the separated amplitude and phase components. $y_{k}^{\prime}(t, \tau)$ is the clipped signal which has been recovered with these components. By means of this step, we can use conventional signal processing software for detection and demodulation without modification.

\section{PERFORMANCE EVALUATION}

We used computer simulation to evaluate the separation performance of our method. Table 1 shows the simulation conditions used. In this evaluation, we assumed that two terminals of different wireless systems each sent the radio signals S1 and S2. The signals' spectrums overlapped each other and their respective power levels were P1 and P2. Their arrival time interval was set randomly in the range of half of the symbol length, whether S1 arrives earlier or later than S2. The channel was set to be an AWGN channel because communications between terminals and flexible access points are usually conducted under the line-of-sight environment in our target system. The relationship of the two overlapped signals is shown in Fig. 4. We defined $\mathrm{S} 1$ as a desired signal and S2 as an interference signal. Figures 5 and 6 show the separation performance obtained with the proposed method and the conventional BPF without signal separation for BPSK and QPSK signals, respectively. In these figures, the horizontal axes are the interval between the center frequencies (shown as $\triangle \mathrm{Fc}$ in Fig. 4), and the vertical axes are the bit error rate (BER) of $\mathrm{S} 1$. When the $\mathrm{P} 1 / \mathrm{P} 2$ ratio is $-3 \mathrm{~dB}$ and the BER is $10^{-3}$, our method improves the interval between center frequencies by $5 \mathrm{kHz}$ for BPSK and by $2 \mathrm{kHz}$ for QPSK signals, as the results shown in Fig. 5 and 6 indicate. Similarly, our method improves the interval between center frequencies, except for the case when the $\mathrm{P} 1 / \mathrm{P} 2$ ratio is $0 \mathrm{~dB}$ and the signals are BPSK signals. In this exceptional case, our method's performance was about the same as that without signal separation. The reason for this is that the reception performance of BPSK signals degrades very little as a result of interference in this case because the interference signal level becomes lower than the desired signal level as the interval between center frequencies becomes wider than $0 \mathrm{~Hz}$. This shows that our method is effective in cases when the performance degrades as a result of interference between overlapped signals.

TABLE I. SIMULATION CONDITIONS.

\begin{tabular}{|c|c|}
\hline Symbol rate & 20 kbauds \\
\hline Sampling rate & $20 \mathrm{Msps}$ \\
\hline FFT points & 8192 \\
\hline Band pass filter & $\begin{array}{c}\text { Root raised cosine filter } \\
\text { (roll-off rate: } 0.5 \text { ) }\end{array}$ \\
\hline Window function & $\begin{array}{c}\text { Raised cosine window } \\
\text { (Window size: } 500 \text { samples) }\end{array}$ \\
\hline Center frequency of sig.1(Fc1) & $1 \mathrm{MHz}$ \\
\hline SNR of sig.1 & 20 dB \\
\hline Channel condition & AWGN without fading \\
\hline
\end{tabular}

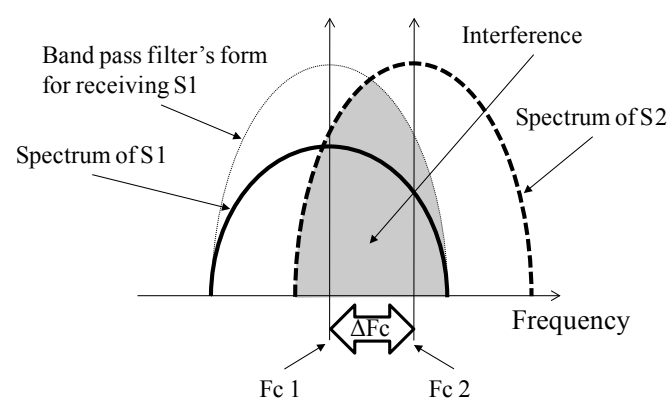

Figure 4. Relationship of two overlapped signals 


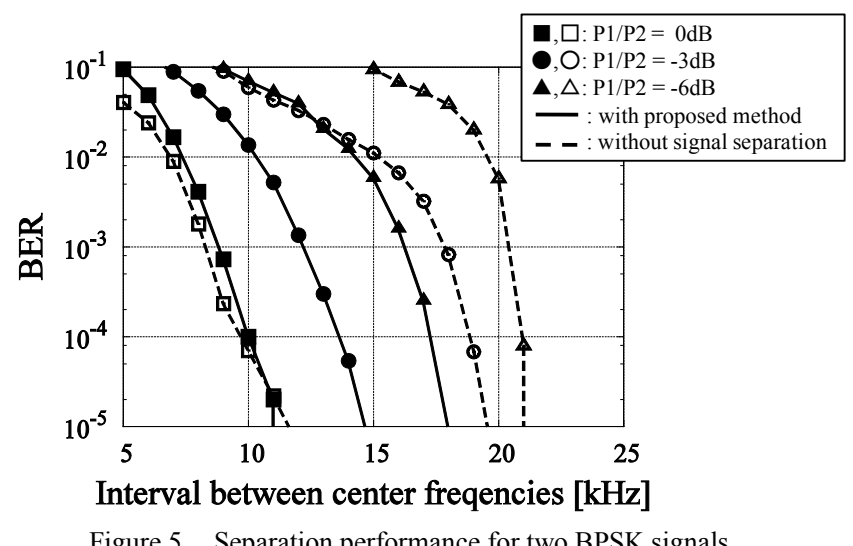

Figure 5. Separation performance for two BPSK signals

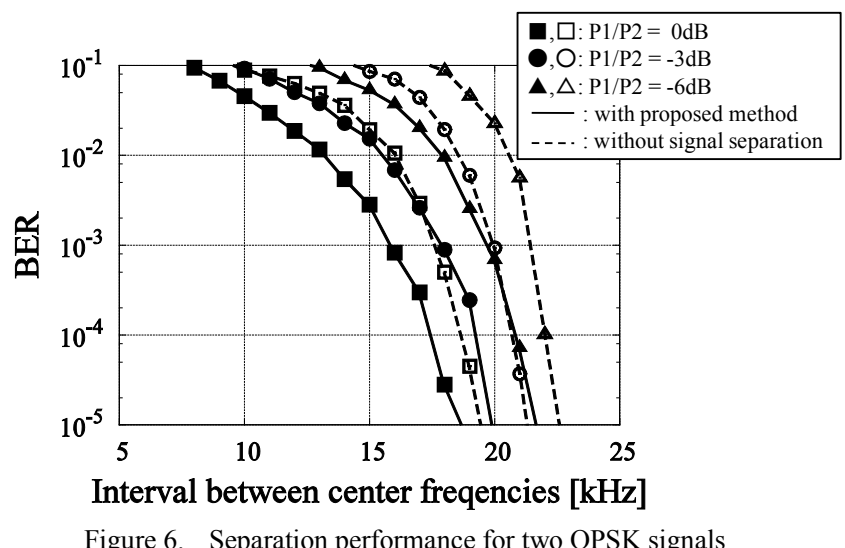

\section{CONCLUSION}

We have described our proposed Flexible Wireless System (FWS), which can flexibly deal with various wireless radio systems. However, its reception performance degrades because of interference caused by simultaneously received multi-system signals. To solve this problem, in this paper we proposed and described a new signal separation method for signal processing with stored radio waves for simultaneously received multisystem signals. The method uses frequency domain information with a wide bandwidth. It separates spectrally overlapped signals through a simple procedure under the assumption that the amplitude and phase components of a variety of modulated signals are constant when clipping wave data with a short time window. It can be adopted even when radio waves are received at an access point with a single antenna. We used computer simulation to evaluate the method's separation performance. In this evaluation, we assumed that two terminals of different wireless systems each sent two spectrally overlapping radio signals. The results show that our method, in comparison to a method in which separation is not used, improves the interval between center frequencies by $5 \mathrm{kHz}$ for BPSK signals and by $2 \mathrm{kHz}$ for QPSK signals when the power ratio of the desired signal and the interference signal is $-3 \mathrm{~dB}$ and the BER is $10^{-3}$. This shows that the method is effective when the reception performance degrades due to interference between overlapped signals. We conclude that our method can improve the frequency utilization efficiency of wireless systems by accepting the spectral overlap for simultaneously received multi-system signals.

\section{REFERENCES}

[1] A. Takahara, H. Hadama, T. Motohashi, and Y. Takigawa, "R\&D Challenges for Future Carrier Networks", NTT Technical Review, No.5, May, 2009.

[2] N. Takahashi, K. Akabane, M. Matsuo, M. Ohta, M. Harada, and K. Okada, "Technologies Towards Networked Objects and Events in the Real World", NTT Technical Review, No. 5, May 2009.

[3] H. Shiba, K. Akabane, T. Yamada, and K. Uehara, "A unified wireless platform architecture for a wide variety of wireless systems," in Proc. SDR'09 Technical Conference, Dec. 2009.

[4] H. Shiomi, T Yata, and Y. Okamura, "Experiment of blind signal separation of wireless mixture using complex valued fast independent component analysis," in Proc. AP-S 2008, pp. 1-4, Jul. 2008.

[5] J. Shang, "A Blind Separation Method for Mixed FSK and QPSK Signals Based on ICA," in Proc. WiCom 2010, pp. 6684-6687, Sept. 2007.

[6] J. Zhuo, C. Sun, Y. Yang, and B. Li, "Joint signal detection and recovery via Blind Signal Separation,” in Proc. WCNIS 2010, pp. 67-71, Jun. 2010.

[7] A. Jourjine, S. Rickard, and Ö. Yilmaz, "Blind separation of disjoint orthogonal signals: Demixing N source from 2 mixtures," in Proc. ICASSP 2000, pp.2985-2988, Jun. 2000.

[8] Ö. Yilmaz and S. Rickard, "Blind separation of speech mixtures via time-frequency masking," IEEE Trans. Signal Processing, vol.52, no.7, pp.1830-1847, Jul. 2004.

[9] A. Blin, S.Araki and S Makino, "Undertermined blind separation of convolutive mixtures of speech using time-frequency mask and mixing matrix estimation," IEICE Trans. Fundamentals, vol.E88-A, no.7, pp.1693-1700, Jul. 2005.

[10] [Online]. Available: http://www.ict-futon.eu/default.aspx

[11] M. Kawashima, Y. Yamaguchi, K. Nishikawa, K. Uehara, "Broadband low noise amplifier with high linearity for software-defined radios,"Proceedings of European Microwave Integrated Circuit Conference (EuMIC) 2007, pp. 243 - 246, October 2007.

[12] T. Yamada, D. Lee, H. Shiba, Y. Yamaguchi, K. Uehara, "A Study on Window Size of Signal Restoration Method for Wide Band Spectrum Block Receiving," IEICE General Conference, B-17-24, Mar. 2010. (in Japanese) 